\title{
THE DIAMETER OF A SCALE-FREE RANDOM GRAPH
}

\author{
BÉLA BOLLOBÁS*, OLIVER RIORDAN
}

\author{
Received August 1, 2000 \\ Revised July 24, 2001
}

\begin{abstract}
We consider a random graph process in which vertices are added to the graph one at a time and joined to a fixed number $m$ of earlier vertices, where each earlier vertex is chosen with probability proportional to its degree. This process was introduced by Barabási and Albert [3], as a simple model of the growth of real-world graphs such as the world-wide web. Computer experiments presented by Barabási, Albert and Jeong [1,5] and heuristic arguments given by Newman, Strogatz and Watts [23] suggest that after $n$ steps the resulting graph should have diameter approximately $\log n$. We show that while this holds for $m=1$, for $m \geq 2$ the diameter is asymptotically $\log n / \log \log n$.
\end{abstract}

\section{Introduction}

Recently there has been considerable interest in studying complex realworld networks and attempting to understand their properties using random graphs as models. Attention has been focused particularly on the 'smallworld' phenomenon, that graphs with a very large number $n$ of vertices often have diameter $\operatorname{around} \log n$. In the context of various standard random graph models, this phenomenon has of course been known for a long time; see for example $[13,14,6]$. That such a small diameter can be expected even when the vertex degrees are constant was shown in [9]. Related results in different contexts include $[19,18,10]$; see also chapter X of [7]. A less standard example showing that when the degrees are constant even a small amount of randomness produces this phenomenon is given in [8]. However, real-world

\footnotetext{
Mathematics Subject Classification (2000): 05C80

* Research supported in part by NSF grant no. DSM 9971788
} 
graphs are often not well approximated by these models, as shown by their degree sequences, for example.

Barabási and Albert [3], as well as several other groups (see [5] and the references therein), noticed that in many real-world examples the fraction $P(k)$ of vertices with degree $k$ follows a power law over a large range, with $P(k)$ proportional to $k^{-\gamma}$ for some constant $\gamma$ independent of the scale of the network. Graphs with this behaviour have been studied in several papers, in particular with respect to how well interconnected they are, as measured by the diameter, or by the average distance between pairs of vertices. Such study has been from one of two points of view. In $[1,5,23]$ graphs are constructed at random subject to the degree sequence having the desired distribution, and the diameter is analyzed (by computer experiments in $[1$, 5], and heuristically in [23]), obtaining an answer of the form $A+B \log n$. This 'small-world phenomenon' is often seen as surprising, as it is in the real world. However, the examples cited in the first paragraph show that in the random graph context if anything is surprising it is that the diameter should be so large; one would expect such a skewed degree sequence to lead to a diameter no larger than, and perhaps smaller than, the $\log n$ obtained for all degrees roughly equal. This is discussed briefly in section 10 .

The above point of view takes the form of the degree sequence as an experimental fact. Putting only this information into the model, one investigates which other properties of the graph (such as small diameter) follow naturally from such a degree sequence, without considering how the degree distribution arises. A different approach is to try to model the growth of the graph in such a way as to explain the distribution of the degrees. In particular, Barabási and Albert suggested modeling such networks using a random graph process with the following description, taken from [3]:

... starting with a small number $\left(m_{0}\right)$ of vertices, at every time step we add a new vertex with $m\left(\leq m_{0}\right)$ edges that link the new vertex to $m$ different vertices already present in the system. To incorporate preferential attachment, we assume that the probability $\Pi$ that a new vertex will be connected to a vertex $i$ depends on the connectivity $k_{i}$ of that vertex, so that $\Pi\left(k_{i}\right)=k_{i} / \sum_{j} k_{j}$. After $t$ steps the model leads to a random network with $t+m_{0}$ vertices and $m t$ edges.

Such a process provides a highly simplified model of the growth of the worldwide web, for example, the vertices representing web sites or web pages, and the edges links from sites to earlier sites, the idea being that a new site is more likely to link to existing sites which are 'popular' (are often linked to) at the time the site is added. It is easy to see heuristically that this process leads to a degree distribution $P(k)$ approximately of the form $P(k) \alpha k^{-3}$ [4], 
so that the number of vertices with degree at least $k$ falls off as $c k^{-2}$ for large $k$. A precise version of this statement will be proved in a forthcoming paper [12]. (Note added: although written later, [12] has appeared first.) Here we shall study the diameter of the resulting graph, showing that it is asymptotically $\log n / \log \log n$ if $m \geq 2$. In contrast, for $m=1$ a result of Pittel [24] states essentially that the diameter is $\Theta(\log n)$. The relationship of these results to previous heuristics is discussed briefly in the final section.

Note that a different type of model leading to the 'small-world phenomenon' was introduced earlier by Watts and Strogatz [28], involving regularly arranged 'local' connections together with random 'global' connections. As the graphs produced have all degrees about the same, the study of the diameter in this case is a separate topic to that of this paper.

\section{The model}

When making the model described in the preceding section precise we have some choice as to how to proceed, since the distribution of a random $m$ element set is not specified by giving the marginal probability that each element is contained in this set. When we add a new vertex to the graph we shall add $m$ new edges one at a time, allowing multiple edges between the same pair of vertices. Also, it will be convenient to allow loops; in terms of the interpretation there is no reason to exclude multiple links from one site to another, or links between different parts of a site or even page.

For precise definitions we start with the case $m=1$. Consider a fixed sequence of vertices $v_{1}, v_{2}, \ldots$. (Most of the time we shall take $v_{i}=i$ to simplify the notation.) We write $d_{G}(v)$ for the degree of the vertex $v$ in the graph $G$. We shall inductively define a random graph process $\left(G_{1}^{t}\right)_{t \geq 0}$ so that $G_{1}^{t}$ is a graph on $\left\{v_{i}: 1 \leq i \leq t\right\}$, as follows. Start with $G_{1}^{0}$, the empty 'graph' with no vertices, or with $G_{1}^{1}$ the graph with one vertex and one loop. Given $G_{1}^{t-1}$, we form $G_{1}^{t}$ by adding the vertex $v_{t}$ together with a single edge between $v_{t}$ and $v_{i}$, where $i$ is chosen randomly with

$$
\mathbb{P}(i=s)= \begin{cases}d_{G_{1}^{t-1}}\left(v_{s}\right) /(2 t-1) & 1 \leq s \leq t-1, \\ 1 /(2 t-1) & s=t .\end{cases}
$$

In other words, we send an edge $e$ from $v_{t}$ to a random vertex $v_{i}$, where the probability that a vertex is chosen as $v_{i}$ is proportional to its degree at the time, counting $e$ as already contributing one to the degree of $v_{t}$. (We shall see why this is convenient later.) For $m>1$ we add $m$ edges from $v_{t}$ one at a time, counting the previous edges as well as the 'outward half' of the edge being added as already contributing to the degrees. Equivalently, we define 
the process $\left(G_{m}^{t}\right)_{t \geq 0}$ by running the process $\left(G_{1}^{t}\right)$ on a sequence $v_{1}^{\prime}, v_{2}^{\prime}, \ldots$; the graph $G_{m}^{t}$ is formed from $G_{1}^{m t}$ by identifying the vertices $v_{1}^{\prime}, v_{2}^{\prime}, \ldots, v_{m}^{\prime}$ to form $v_{1}$, identifying $v_{m+1}^{\prime}, v_{m+2}^{\prime}, \ldots, v_{2 m}^{\prime}$ to form $v_{2}$, and so on.

From now on we shall take $v_{i}=i$, so $G_{m}^{t}$ is a graph on $[t]=\{1,2, \ldots, t\}$. We have defined $G_{m}^{t}$ as an undirected graph, and it is as an undirected graph that we shall measure its diameter. We note, however, that $G_{m}^{t}$ has a very natural orientation: direct each edge $i j$ with $i>j$ from $i$ to $j$, so each nonloop edge is directed from a later vertex to an earlier one. One can check that if any possible graph $G_{m}^{t}$ is given without the vertex labels, the edge orientations can be uniquely reconstructed. As we shall always treat $G_{m}^{t}$ as a graph with labelled vertices, this is of no relevance here.

Often we shall consider not the whole process, but just the graph obtained at one particular time: we shall write $\mathcal{G}_{m}^{n}$ for the probability space of graphs on $[n]$ where a random $G_{m}^{n} \in \mathcal{G}_{m}^{n}$ has the distribution derived from the process above.

As the process $\left(G_{m}^{t}\right)$ is defined in terms of $\left(G_{1}^{t}\right)$ we can deduce the properties of a random $G_{m}^{n} \in \mathcal{G}_{m}^{n}$ from those of a random $G_{1}^{m n} \in \mathcal{G}_{1}^{m n}$. For the moment we shall thus restrict our attention to the case $m=1$. The reason for allowing loops is that this allows us to give a precise alternative description of the distribution of $G_{1}^{n}$, in terms of pairings.

An $n$-pairing is a partition of the set $\{1,2, \ldots, 2 n\}$ into pairs, so there are $(2 n) ! /\left(n ! 2^{n}\right) n$-pairings. These objects are sometimes thought of as linearized chord diagrams (or $L C D s$ ) $[26,11]$, where an LCD with $n$ chords consists of $2 n$ distinct points on the $x$-axis paired off by semi-circular chords in the upper half plane. Two LCDs are considered to be the same when one can be turned into the other by moving the points on the $x$-axis without changing their order. Thinking of pairings as LCDs, we shall talk of chords and their left and right endpoints. We form a graph $\phi(L)$ from an LCD $L$ as follows: starting from the left, identify all endpoints up to and including the first right endpoint reached to form vertex 1 . Then identify all further endpoints up to the next right endpoint to form vertex 2, and so on. For the edges, replace each chord by an edge joining the vertex corresponding to its right endpoint to that corresponding to its left endpoint. We claim that if $L$ is chosen uniformly at random from all $(2 n) ! /\left(n ! 2^{n}\right)$ LCDs with $n$ chords (i.e., $n$-pairings), then $\phi(L)$ has the same distribution as a random $G_{1}^{n} \in \mathcal{G}_{1}^{n}$. To see this note that $L$ can be obtained by taking a random LCD $L^{\prime}$ with $n-1$ chords and adding a new chord whose right endpoint is to the right of all $n-1$ chords, and whose left endpoint lies in one of the $2 n-1$ possible places, each chosen with equal probability. This corresponds to adding a new vertex 
to $\phi\left(L^{\prime}\right)$ and joining it to another vertex with probabilities according to the degrees, exactly as in the description of $\left(G_{1}^{n}\right)$.

We now study the diameter of $G_{m}^{n}$ as an undirected graph, using this description from pairings.

\section{Results}

Our aim is to prove the following result, where $G_{m}^{n} \in \mathcal{G}_{m}^{n}$ is the random graph described above. Following standard terminology we shall say that almost every or a.e. $G_{m}^{n} \in \mathcal{G}_{m}^{n}$ has a certain property if the probability that a random $G_{m}^{n} \in \mathcal{G}_{m}^{n}$ has this property tends to 1 as $n \rightarrow \infty$, keeping $m$ fixed.

Theorem 1. Fix an integer $m \geq 2$ and a positive real number $\epsilon$. Then a.e. $G_{m}^{n} \in \mathcal{G}_{m}^{n}$ is connected and has diameter $\operatorname{diam}\left(G_{m}^{n}\right)$ satisfying

$$
(1-\epsilon) \log n / \log \log n \leq \operatorname{diam}\left(G_{m}^{n}\right) \leq(1+\epsilon) \log n / \log \log n .
$$

The lower bound is relatively easy to prove since we can proceed directly from the definition of the process $\left(G_{m}^{t}\right)$. This bound is proved in section 4 , in a tighter form than that given above. Our proof of the upper bound is much more complicated; for this we use an alternative method for generating a random $G_{m}^{n}$ based on generating a random $m n$-pairing in a certain way, described in section 6 .

In the rest of the paper we shall use the following standard notation. Given a graph $G$ we shall write $\Delta(G)$ for its maximum degree, $E(G)$ for the set of its edges, and $e(G)=|E(G)|$ for the number of edges. We write $\operatorname{Bi}(n, p)$ for the binomial distribution with parameters $n$ and $p$, and $\mathbb{I}_{A}$ for the indicator function of an event $A$. All logarithms are natural unless otherwise indicated.

\section{The lower bound}

To prove the lower bound in Theorem 1 we shall consider $G_{1}^{N}$ with $N=m n$. We shall compare $G_{1}^{N}$ with a random graph in which every edge $i j$ is present with probability $C / \sqrt{i j}$ independently of the other possible edges, for some constant $C$. It turns out that 'small' graphs are not much more likely to occur in $G_{1}^{N}$ than in this random graph, despite the dependence present in $G_{1}^{N}$.

Recall that $G_{1}^{N}$ is defined as a graph on $[N]=\{1,2, \ldots, N\}$. We shall write $g_{j}$ for the vertex to which vertex $j$ sends an edge, so $E\left(G_{1}^{j}\right)=E\left(G_{1}^{j-1}\right) \cup\left\{g_{j} j\right\}$. 
Now given $G_{1}^{j-1}$, the probability that $g_{j}=i$ is proportional to the degree of $i$ in $G_{1}^{j-1}$. Thus, given that $g_{j}=i$, the vertex $i$ is likely to have a relatively large degree in $G_{1}^{j-1}$, and hence in $G_{1}^{t}$ for all $t$. This suggests that the events $g_{j}=i$ and $g_{j^{\prime}}=i^{\prime}$ are positively correlated if $i=i^{\prime}$, and negatively correlated otherwise. However, as we shall now see, for $i=i^{\prime}$ any positive correlation is not too strong. Later we shall prove the negative correlation in a more general context. All constants implicit in the $O()$ terms are absolute constants.

Lemma 2. If $1 \leq i<j$, then

$$
\mathbb{P}\left(g_{j}=i\right)=O\left((i j)^{-1 / 2}\right) .
$$

Also, if $1 \leq i<j<k$, then

$$
\mathbb{P}\left(g_{j}=i, g_{k}=i\right)=O\left(i^{-1}(j k)^{-1 / 2}\right) .
$$

Proof. We shall consider the process $\left(G_{1}^{t}\right)$, applying repeatedly the fact that $\mathbb{E}(A)=\mathbb{E}(\mathbb{E}(A \mid B))$, with $B$ the random variable $G_{1}^{t-1}$. Let $d_{t, i}=d_{G_{1}^{t}}(i)$ be the degree of the vertex $i$ in the graph $G_{1}^{t}$. We start by evaluating the expectation of $d_{t, i}$ for all $t$. From the definition of the process $\left(G_{1}^{t}\right)$ we have

$$
\mathbb{P}\left(g_{t}=i \mid G_{1}^{t-1}\right)= \begin{cases}d_{t-1, i} /(2 t-1) & t>i \\ 1 /(2 i-1) & t=i .\end{cases}
$$

For $t>i$ we have $d_{t, i}=d_{t-1, i}+\mathbb{I}_{\left\{g_{t}=i\right\}}$, so

$$
\mathbb{E}\left(d_{t, i} \mid G_{1}^{t-1}\right)=d_{t-1, i}+\frac{d_{t-1, i}}{2 t-1}=\left(1+\frac{1}{2 t-1}\right) d_{t-1, i} .
$$

Taking expectations of both sides,

$$
\mathbb{E}\left(d_{t, i}\right)=\left(1+\frac{1}{2 t-1}\right) \mathbb{E}\left(d_{t-1, i}\right) .
$$

Let us write $\mu_{t, i}$ for $\mathbb{E}\left(d_{t, i}\right)$. Then, as $\mu_{i, i}=2 i /(2 i-1)$, we have for all $t \geq i$ that

$$
\mu_{t, i}=\prod_{s=i}^{t}\left(1+\frac{1}{2 s-1}\right)=O(\sqrt{t / i}),
$$

where the estimate follows by taking logarithms and comparing the resulting sum with an integral. Taking the expectation of both sides of (3) for $t=j>i$ we obtain

$$
\mathbb{P}\left(g_{j}=i\right)=\mu_{j-1, i} /(2 j-1)=O\left((i j)^{-1 / 2}\right)
$$


proving the first part of the lemma.

For the second statement it turns out that we need to consider second moments. Suppose that $t>i$. Then from (3) we have

$$
\begin{aligned}
\mathbb{E}\left(d_{t, i}^{2} \mid G_{1}^{t-1}\right) & =d_{t-1, i}^{2}\left(1-\frac{d_{t-1, i}}{2 t-1}\right)+\left(d_{t-1, i}+1\right)^{2} \frac{d_{t-1, i}}{2 t-1} \\
& =d_{t-1, i}^{2}\left(1+\frac{2}{2 t-1}\right)+\frac{d_{t-1, i}}{2 t-1} .
\end{aligned}
$$

Taking the expectation of both sides we obtain

$$
\mathbb{E}\left(d_{t, i}^{2}\right)=\left(1+\frac{2}{2 t-1}\right) \mathbb{E}\left(d_{t-1, i}^{2}\right)+\frac{\mathbb{E}\left(d_{t-1, i}\right)}{2 t-1} .
$$

Adding (4) and (5), and writing $\mu_{t, i}^{(2)}$ for $\mathbb{E}\left(d_{t, i}^{2}\right)+\mathbb{E}\left(d_{t, i}\right)$, we obtain

$$
\mu_{t, i}^{(2)}=\left(1+\frac{2}{2 t-1}\right) \mu_{t-1, i}^{(2)},
$$

while $\mu_{i, i}^{(2)}=O(1)$ as $d_{i, i}$ is either 1 or 2 . Hence

$$
\mu_{t, i}^{(2)}=\prod_{s=i+1}^{t}\left(1+\frac{2}{2 s-1}\right) \mu_{i, i}^{(2)}=\frac{2 t+1}{2 i+1} \mu_{i, i}^{(2)}=O(t / i) .
$$

Now from (3) we have

$$
\mathbb{E}\left(d_{j, i} \mathbb{I}_{\left\{g_{j}=i\right\}} \mid G_{1}^{j-1}\right)=\left(d_{j-1, i}+1\right) \frac{d_{j-1, i}}{2 j-1} .
$$

Taking the expectation of both sides,

$$
\mathbb{E}\left(d_{j, i} \mathbb{I}_{\left\{g_{j}=i\right\}}\right)=\frac{\mu_{j-1, i}^{(2)}}{2 j-1}=O\left(i^{-1}\right) .
$$

Arguing as in the proof of (1) above, for all $t>j$ we have

$$
\mathbb{E}\left(d_{t, i} \mathbb{I}_{\left\{g_{j}=i\right\}}\right)=O(\sqrt{t / j}) \mathbb{E}\left(d_{j, i} \mathbb{I}_{\left\{g_{j}=i\right\}}\right)=O\left(i^{-1} \sqrt{t / j}\right) .
$$

Now

$$
\mathbb{P}\left(g_{j}=i, g_{k}=i \mid G_{1}^{k-1}\right)=\mathbb{I}_{\left\{g_{j}=i\right\}} \frac{d_{k-1, i}}{2 k-1} .
$$

Taking expectations gives

$$
\mathbb{P}\left(g_{j}=i, g_{k}=i\right)=O\left((i k)^{-1} \sqrt{k / j}\right)=O\left(i^{-1}(j k)^{-1 / 2}\right),
$$


completing the proof of the lemma.

Note that a result similar to the above can be proved for the probability that $g_{j_{1}}=g_{j_{2}}=\cdots=g_{j_{r}}=i$, where $r$ is fixed and $i<j_{1}<\cdots<j_{r}$. In fact, our proof actually gives a formula this probability for $r=1,2$. Since this is rather cumbersome, it is more convenient to use the estimate stated in the lemma.

We now turn to the negative correlation case. For this we shall use the following alternative description of the process $\left(G_{1}^{t}\right)$. We consider $G_{1}^{t}$ as consisting of $2 t$ half-edges numbered $1,2, \ldots, 2 t$, each attached to a vertex from $[t]$. Given $G_{1}^{t-1}$, we obtain $G_{1}^{t}$ by adding a new half-edge (number $2 t-1$ ) attached to vertex $t$, and then a half-edge (number $2 t$ ) which must be attached to each vertex with probability proportional to its degree. As the degree of a vertex is the number of half-edges already attached to it, we can think of half-edge $2 t$ as being associated to one of the $2 t-1$ existing half-edges chosen uniformly at random, and attached to whichever vertex this half-edge is attached to. Let us write $h_{t}$ for the number of the half-edge to which half-edge $2 t$ is attached, so the $h_{t}$ are independent, with $h_{t}$ chosen uniformly from $\{1,2, \ldots, 2 t-1\}$.

Lemma 3. Let $E$ and $E^{\prime}$ be events of the form

$$
E=\bigcap_{s=1}^{r}\left\{g_{j_{s}}=i_{s}\right\}, \quad E^{\prime}=\bigcap_{s=1}^{r^{\prime}}\left\{g_{j_{s}^{\prime}}=i_{s}^{\prime}\right\},
$$

where $i_{s}<j_{s}, i_{s}^{\prime}<j_{s}^{\prime}$ for all s. If the sets $\left\{i_{1}, \ldots, i_{r}\right\}$ and $\left\{i_{1}^{\prime}, \ldots, i_{r^{\prime}}^{\prime}\right\}$ are disjoint then

$$
\mathbb{P}\left(E \cap E^{\prime}\right) \leq \mathbb{P}(E) \mathbb{P}\left(E^{\prime}\right) .
$$

Proof. In terms of the description above, $g_{j}=i$ if and only if either $h_{j}=2 i-1$, or for some $j^{\prime}$ we have $h_{j}=2 j^{\prime}, h_{j^{\prime}}=2 i-1$, or for some $j^{\prime}, j^{\prime \prime} \ldots$. More formally, the event $g_{j}=i$ is a disjoint union of events of the form

$$
\exists s \geq 0, j_{1}, \ldots, j_{s} \text { s.t. }(\forall a: 0 \leq a<s) h_{j_{a}}=2 j_{a+1}, h_{j_{s}}=2 i-1,
$$

where $j_{0}=j$. Thus we can write $E$ as a disjoint union of events $F_{k}$, each an intersection of events of the form (6). Similarly we can write $E^{\prime}$ as a disjoint union of such events $F_{l}^{\prime}$. Now for any $k, l$ the events $F_{k}$ and $F_{l}^{\prime}$ are either independent, if no $h_{t}$ appears in both, or inconsistent; if some $h_{t}$ appears in both, the corresponding half-edge must be attached to one of the vertices $i_{1}, \ldots, i_{r}$ if $F_{k}$ holds, but to one of the vertices $i_{1}^{\prime} \ldots, i_{r^{\prime}}^{\prime}$ if $F_{l}^{\prime}$ holds. Thus $\mathbb{P}\left(F_{k} \cap F_{l}^{\prime}\right) \leq \mathbb{P}\left(F_{k}\right) \mathbb{P}\left(F_{l}^{\prime}\right)$ in all cases. Summing over $k$ and $l$ completes the proof of the lemma. 
Combining the two lemmas above gives an upper bound on the probability that a simple enough graph is contained in $G_{1}^{N}$. In what follows, the subgraph $S$ we are looking for is a graph on the same vertex set as $G_{1}^{N}$. To say that $S$ is a subgraph of $G_{1}^{N}$, or occurs in $G_{1}^{N}$, is thus to say that the pairs of vertices joined in $S$ are joined in $G_{1}^{N}$, not that $G_{1}^{N}$ has a subgraph isomorphic to $S$. A vertex $i$ is earlier than a vertex $j$ if $i<j$, and later if $i>j$.

Lemma 4. Let $S$ be a loopless graph on $[N]$ in which each vertex is joined to at most one earlier vertex and at most two later vertices. Then

$$
\mathbb{P}\left(S \subset G_{1}^{N}\right) \leq C^{e(S)} \prod_{i j \in E(S)} \frac{1}{\sqrt{i j}},
$$

for some absolute constant $C$.

Proof. Grouping the edges of $S$ according to the earlier vertex of each, the event $S \subset G_{1}^{N}$ is the intersection of events

$$
E_{k}=\left\{g_{j_{k, 1}}=\cdots=g_{j_{k, n_{k}}}=i_{k}\right\},
$$

where the $i_{k}$ are distinct and $n_{k}=1,2$ for all $k$. By Lemma 2 there is an absolute constant $C$ such that

$$
\mathbb{P}\left(E_{k}\right) \leq C^{n_{k}} \prod_{l=1}^{n_{k}}\left(i_{k} j_{k, l}\right)^{-1 / 2}
$$

for every $k$. Also, by Lemma 3 , the events $E_{k}$ and $E_{1} \cap \cdots \cap E_{k-1}$ are negatively correlated for every $k$, so

$$
\mathbb{P}\left(S \subset G_{1}^{N}\right)=\mathbb{P}\left(\bigcap_{k} E_{k}\right) \leq \prod_{k} \mathbb{P}\left(E_{k}\right) .
$$

Combined with the bound on $\mathbb{P}\left(E_{k}\right)$ above this completes the proof.

Note that we did not use the condition that $S$ is loopless; this was taken for simplicity. A more general version of Lemma 2 would of course give a more general version of Lemma 4.

Recalling that $G_{m}^{n}$ is obtained by identifying the vertices of $G_{1}^{m n}$ in groups of $m$, we can deduce the lower bound in Theorem 1 in the following more precise form.

Theorem 5. Let $m \geq 1$ be fixed. Then $\operatorname{diam}\left(G_{m}^{n}\right)>\log n / \log \left(3 C m^{2} \log n\right)$ for a.e. $G_{m}^{n} \in \mathcal{G}_{m}^{n}$, where $C$ is the constant in Lemma 4. 
Proof. We shall prove slightly more, namely that with probability tending to 1 the distance $\rho(n, n-1)$ between the vertices $n$ and $n-1$ of $G_{m}^{n}$ is greater than $L=\log n / \log \left(3 C m^{2} \log n\right)$, with $C$ as above.

Consider a particular path $P=v_{0} v_{1} \cdots v_{l}$ on $[n]$ of length $l \leq L$. A graph $S$ on $[m n]$ is a realization of $P$ if $S$ consists of edges $x_{t} y_{t+1}, t=0,1, \ldots, l-1$, with $\left\lceil x_{t} / m\right\rceil=\left\lceil y_{t} / m\right\rceil=v_{t}$. As $P$ and hence $S$ has maximum degree at most two, Lemma 4 tells us that one such realization $S$ is present in $G_{1}^{m n}$ with probability at most

$$
C^{l} \prod_{t=0}^{l-1} \frac{1}{\sqrt{x_{t} y_{t+1}}} \leq C^{l} \prod_{t=0}^{l-1} \frac{1}{\sqrt{v_{t} v_{t+1}}}=\frac{C^{l}}{\sqrt{v_{0} v_{l}}} \prod_{t=1}^{l-1} \frac{1}{v_{t}} .
$$

As there are exactly $m^{2 l}$ realizations of $P$, and $P \subset G_{m}^{n}$ if and only if at least one is present in $G_{1}^{m n}$, we have

$$
\mathbb{P}\left(P \subset G_{m}^{n}\right) \leq \frac{\left(C m^{2}\right)^{l}}{\sqrt{v_{0} v_{l}}} \prod_{t=1}^{l-1} \frac{1}{v_{t}} .
$$

Thus, if $n$ is large enough, the expected number of paths of length $l$ between the vertices $n$ and $n-1$ of $G_{m}^{n}$ is bounded by

$$
\begin{aligned}
\frac{\left(C m^{2}\right)^{l}}{\sqrt{n(n-1)}} \sum_{1 \leq v_{1}, \ldots, v_{l-1} \leq n} \prod_{t=1}^{l-1} \frac{1}{v_{t}} & =\frac{\left(C m^{2}\right)^{l}}{\sqrt{n(n-1)}}\left(\sum_{v=1}^{n} \frac{1}{v}\right)^{l-1} \\
& \leq \frac{\left(2 C m^{2}\right)^{l}}{n}(\log n)^{l-1} \\
& \leq(2 / 3)^{l}(\log n)^{-1}
\end{aligned}
$$

as $l \leq L$ implies that $\left(3 C m^{2} \log n\right)^{l} \leq n$. Summing over $1 \leq l \leq L$, we find that the expected number of paths of length at most $L$ joining the vertices $n$ and $n-1$ tends to zero, so $\rho(n, n-1)>L$ in a.e. $G_{m}^{n}$.

This proves the lower bound in Theorem 1 . In the following sections we prove the upper bound for $m \geq 2$.

\section{The upper bound}

As the proof is rather lengthy, we give an outline before turning to the details. The basic idea is to use the random pairing model from section 2 . Considering the pairing as an LCD pairing of independent uniformly chosen random points in $[0,1]$, we shall choose the right endpoint of each chord first, according to the appropriate (non-uniform) distribution. Given these right 
endpoints, the left endpoints are independent of one another, each being constrained to come before its corresponding right endpoint. (We may of course ignore the probability 0 event that two endpoints coincide.) We shall first show that the distribution of right endpoints is likely to be 'nice', i.e., to have certain uniformity properties. For the rest of the proof we then fix a 'nice' distribution of right endpoints and work with this.

The main idea of the proof is to start with a given vertex $v$, and consider the set $N_{k}(v)$ of vertices within distance $k$ of $v$, aiming to show that this increases in size at a certain rate. Rather than size, it turns out that one should consider 'weight', assigning weight $i^{-1 / 2}$ to the $i^{\text {th }}$ vertex. Essentially, given the weight $w\left(N_{k-1}\right)$ of $N_{k-1}$, the expected value of $w\left(N_{k}\right)$ is $(\log n) w\left(N_{k-1}\right)$, as long as this is not too large. Thus in $\Theta(\log n / \log \log n)$ steps from $v$ one can reach many vertices. We complete the proof by showing that from these many vertices one can almost certainly reach the first vertex in one step. Unfortunately there are several complications.

One essential complication is that when $w\left(N_{k-1}\right)$ is fairly small, although $w\left(N_{k}\right)$ has expectation $(\log n) w\left(N_{k-1}\right)$, it has a very skew distribution, and is almost always much smaller than its mean. This means that we have to work with a 'blow-up factor' which starts off as a constant and increases as we proceed.

Another complication is that the main argument we use does not work starting from a single vertex near the end. To deal with this we show separately that all such vertices are likely to be joined by short paths to sufficiently early vertices.

Finally, the 'nice' distributions of right endpoints are not really all that nice; globally they behave exactly as we would like, but it is impossible to avoid some local variation. This complicates the details of the proof.

The main probabilistic tool we shall use in the rest of the paper is the following lemma given by Janson [17], which may be deduced from the Chernoff bounds [15].

Lemma 6. Let $X=X_{1}+\cdots+X_{k}$ where the $X_{i}$ are independent Bernoulli random variables with $\mathbb{P}\left(X_{i}=1\right)=p_{i}$. Let $\mu=\mathbb{E} X=p_{1}+\cdots+p_{k}$. Then for $t \geq 0$ we have

$$
\mathbb{P}(X \geq \mu+t) \leq \exp \left(-\frac{t^{2}}{2(\mu+t / 3)}\right)
$$

and

$$
\mathbb{P}(X \leq \mu-t) \leq \exp \left(-\frac{t^{2}}{2 \mu}\right)
$$




\section{Pairings on $[0,1]$}

We start by describing the method we shall use to generate the random graph $G_{m}^{n}$. Using the results of section 2, this is equivalent to generating a random pairing of the integers $\{1,2, \ldots, 2 N\}$, where $N=m n$.

Let $x_{1}, \ldots, x_{2 N}$ be $2 N$ independent samples from the uniform distribution on $[0,1]$. Assuming that these $2 N$ points are distinct, our LCD (linearized chord diagram) is given by pairing $x_{2 i-1}$ with $x_{2 i}$ for each $i$. Relabelling the $x_{i}$ as $1,2, \ldots$ in ascending order, this gives a random pairing with the correct distribution. To see this note that, loosely speaking, for any set $\left\{x_{1}, \ldots, x_{2 N}\right\}$ of $2 N$ distinct elements of $[0,1]$, all $(2 N)$ ! possibilities for the order in which $x_{1}, \ldots, x_{2 N}$ take these values are equally likely.

We now consider generating a pairing starting with the right endpoints. We call a random variable with density function $2 x, 0<x<1$, an $M_{2}(0,1)$ random variable. Let $\left\{l_{i}, r_{i}\right\}=\left\{x_{2 i-1}, x_{2 i}\right\}$ with $l_{i}<r_{i}$. As $\mathbb{P}\left(\max \left\{x_{2 i-1}, x_{2 i}\right\} \leq t\right)=t^{2}$, the $r_{i}$ are independent $M_{2}(0,1)$ random variables. Also, given $r_{1}, \ldots, r_{N}$, the random variables $l_{1}, \ldots, l_{N}$ are independent with $l_{i}$ uniformly distributed on $\left[0, r_{i}\right]$. To obtain the pairing we must sort all the endpoints together. We shall first sort the right endpoints, and then consider between which right endpoint each left endpoint lies. Our first lemma concerns properties of the sorted right endpoints.

For the rest of this section set $N=m n$. Let $r_{1}, \ldots, r_{N}$ be independent $M_{2}(0,1)$ random variables, and let $R_{1}, \ldots, R_{N}$ be obtained by sorting $r_{1}, \ldots, r_{N}$ into ascending order. When it comes to constructing the actual graph $G_{m}^{n}$, we shall only be interested in every $m^{\text {th }}$ endpoint and the spacings between them, so for $1 \leq i \leq n$ let $W_{i}=R_{m i}$, and let $w_{i}=W_{i}-W_{i-1}$, taking $W_{0}=0$. We shall show that, in a certain range, the $W_{i}$ have the values one might expect. For the $w_{i}$ we can only say that this holds on average over certain intervals.

For the rest of the paper we write $s=2^{a}$ for the smallest power of 2 larger than $(\log n)^{7}$, and $2^{b}$ for the largest power of 2 smaller than $2 n / 3$. Note that $a<b$ if $n$ is large enough. We shall consider the intervals $I_{t}=\left[2^{t}+1,2^{t+1}\right]$, for $a \leq t<b$.

Lemma 7. Let $m \geq 2$ be fixed. Using the definitions above, each of the following five events has probability tending to 1 as $n \rightarrow \infty$ :

$$
\begin{gathered}
E_{1}=\left\{\left|W_{i}-\sqrt{\frac{i}{n}}\right| \leq \frac{1}{10} \sqrt{\frac{i}{n}} \text { for } s \leq i \leq n\right\}, \\
E_{2}=\left\{I_{t} \text { contains at least } 2^{t-1} \text { vertices } i \text { with } w_{i} \geq \frac{1}{10 \sqrt{i n}}, a \leq t<b\right\},
\end{gathered}
$$




$$
\begin{gathered}
E_{3}=\left\{w_{1} \geq \frac{4}{\log n \sqrt{n}}\right\}, \\
E_{4}=\left\{w_{i} \geq \frac{(\log n)^{2}}{n} \text { for } i<n^{1 / 5}\right\}, \\
E_{5}=\left\{w_{i} \leq n^{-4 / 5} \text { for } i>n /(\log n)^{5}\right\} .
\end{gathered}
$$

The events $E_{1}$ and $E_{2}$ describe the main properties of the $W_{i}$ and $w_{i}$ we shall use. The remaining properties are simple technicalities we shall need in section 7 . In the following proof, and in the rest of the paper, we shall always assume that $n$ is larger than some sufficiently large constant, even when this is not explicitly stated.

Proof of Lemma 7. Let us write $R(x)$ for $\left|\left\{i: r_{i} \leq x\right\}\right|=\left|\left\{i: R_{i} \leq x\right\}\right|$. For $0 \leq x \leq 1$ we have $R(x) \sim \operatorname{Bi}\left(m n, x^{2}\right)$. As $W_{i}=R_{m i}$, we have that $W_{i} \leq x$ if and only if $R(x) \geq m i$.

Let $x=\frac{9}{10} \sqrt{i / n}$. Then as $\mathbb{E} R(x)=m n x^{2}=81 m i / 100$, Lemma 6 implies that

$$
\mathbb{P}\left(W_{i} \leq x\right)=\mathbb{P}(R(x) \geq m i) \leq e^{-m i / 60} .
$$

Similarly, with $x=\frac{11}{10} \sqrt{i / n}$ either $x \geq 1$ or $\mathbb{E} R(x)=121 \mathrm{mi} / 100$, and in either case

$$
\mathbb{P}\left(W_{i}>x\right)=\mathbb{P}(R(x)<m i) \leq e^{-m i / 60}
$$

Thus

$$
\mathbb{P}\left(E_{1}^{c}\right) \leq 2 \sum_{i=s}^{\infty} e^{-i / 60}=o(1)
$$

since $s \rightarrow \infty$ as $n \rightarrow \infty$.

It now suffices to show that $\mathbb{P}\left(E_{r}^{c} \cap E_{1}\right)=o(1)$ for $r=2, \ldots, 5$.

Given $R_{m i}$ and the set $\mathcal{J}$ of indices $j$ for which $r_{j}>R_{m i}$, each of the $m(n-$ i) variables $r_{j}, j \in \mathcal{J}$, is independently distributed with density $2 x /\left(1-R_{m i}^{2}\right)$, $R_{m i}<x<1$. For $R_{m(i+1)}=R_{m i+m}$ to be less than $R_{m i}+y$ it must happen that at least $m$ of these $r_{j}$ fall in the interval $\left[R_{m i}, R_{m i}+y\right]$. Conditional on $R_{m i}$, the expected number of $r_{j}$ in this interval is exactly

$$
m(n-i) \frac{\left(R_{m i}+y\right)^{2}-R_{m i}^{2}}{1-R_{m i}^{2}} .
$$


Thus

$$
\mathbb{P}\left(R_{m(i+1)} \leq R_{m i}+y \mid R_{m i}\right) \leq(n-i) \frac{\left(R_{m i}+y\right)^{2}-R_{m i}^{2}}{1-R_{m i}^{2}} .
$$

Translating this to a statement about the $W_{i}$, and replacing $i$ by $i-1$ for convenience later,

$$
\mathbb{P}\left(w_{i} \leq y \mid W_{i-1}\right) \leq(n-i+1) \frac{\left(W_{i-1}+y\right)^{2}-W_{i-1}^{2}}{1-W_{i-1}^{2}} .
$$

For $s+1 \leq i \leq 2 n / 3$, provided $W_{i-1} \leq \frac{11}{10} \sqrt{\frac{i-1}{n}}$ this probability is at most $2 n\left(2.2 y \sqrt{i / n}+y^{2}\right)$. Thus, for $i$ in this range,

$$
\mathbb{P}\left(w_{i}<\frac{1}{10 \sqrt{i n}} \mid W_{i-1}\right) \leq \frac{9}{20}
$$

when $W_{i-1} \leq \frac{11}{10} \sqrt{\frac{i-1}{n}}$. As $W_{i-1}$ satisfies this condition when $E_{1}$ holds, writing $B_{i}$ for the 'bad' event that $w_{i}<\frac{1}{10 \sqrt{\text { in }}}$ we have

$$
\mathbb{P}\left(B_{i} \cap E_{1} \mid W_{i-1}\right) \leq \frac{9}{20}
$$

on the whole probability space.

Now, given $W_{s}$ and $w_{s+1}, \ldots, w_{i-1}$, the distribution of $w_{i}$ depends only on $W_{i-1}=W_{s}+w_{s+1}+\cdots+w_{i-1}$. Thus, if for $i=s+1, \ldots, 2^{b}$ we examine the $w_{i}$ in turn, at each stage the conditional probability of $B_{i} \cap E_{1}$ is at most $9 / 20$. Restricting our attention to $i \in I_{t}$ for some $a \leq t<b$, it follows that the quantity

$$
Z_{t}=\left|\left\{i \in I_{t}: w_{i}<\frac{1}{10 \sqrt{i n}}\right\}\right| \times \mathbb{I}_{E_{1}}
$$

is stochastically dominated by a $\operatorname{Bi}\left(2^{t}, 9 / 20\right)$ distribution, and so from Lemma 6

$$
\mathbb{P}\left(Z_{t}>2^{t-1}\right) \leq \exp \left(-2^{t} / 400\right) .
$$

As $E_{2}^{c} \cap E_{1}$ is exactly the event that for some $a \leq t<b$ we have $Z_{t}>2^{t-1}$,

$$
\mathbb{P}\left(E_{2}^{c} \cap E_{1}\right) \leq \sum_{t=a}^{b-1} \exp \left(-2^{t} / 400\right)=o(1),
$$

as $a \rightarrow \infty$. 
Turning to $E_{3}$, note that $w_{1}=W_{1}$. Taking $W_{0}$ to be identically zero, the argument giving (7) applies with $i=1$ to give $\mathbb{P}\left(w_{1} \leq x\right) \leq n x^{2}$ and hence $\mathbb{P}\left(E_{3}\right)=1-o(1)$.

Suppose that $E_{4}^{c} \cap E_{1}$ holds, and let $\delta=(\log n)^{2} / n$. Then as $E_{1}$ holds we have $W_{\left\lfloor n^{1 / 5}\right\rfloor} \leq 2 n^{-2 / 5}$. As $E_{4}$ does not hold it follows that some interval $[x, x+\delta]$ with $0 \leq x<2 n^{-2 / 5}$ contains two of the $W_{i}$, and hence two of the $r_{i}$. Each such interval is contained in one of the intervals $J_{t}=[t \delta,(t+2) \delta]$, $0 \leq t \leq n^{3 / 5}$. The probability that a particular $r_{i}$ lies in one such $J_{t}$ is exactly $(4 t+4) \delta^{2}$, so the probability that at least two $r_{i}$ lie in $J_{t}$ is at most $m n(m n-1)(4 t+4)^{2} \delta^{4} / 2<(\log n)^{9}(t+1)^{2} / n^{2}$. Thus

$$
\mathbb{P}\left(E_{4}^{c} \cap E_{1}\right) \leq \sum_{t=0}^{n^{3 / 5}}(\log n)^{9}(t+1)^{2} / n^{2}=o(1),
$$

so $\mathbb{P}\left(E_{4}\right)=1-o(1)$.

Finally, suppose that $E_{5}^{c} \cap E_{1}$ holds. This time let $\delta=n^{-4 / 5}$. Then for some $x,(\log n)^{-3}<x<1-\delta$, the interval $[x, x+\delta]$ contains no $W_{i}$, and hence contains at most $m-1$ of the $r_{i}$. Setting $\delta^{\prime}=\delta /(m+1)$, each such interval contains $m$ disjoint intervals of the form $\left[t \delta^{\prime},(t+1) \delta^{\prime}\right)$ with $t$ an integer and $(\log n)^{-3}<t \delta^{\prime}<1-\delta^{\prime}$, one of which must contain no $r_{i}$. For a given $t$, the number of $r_{i}$ in $\left[t \delta^{\prime},(t+1) \delta^{\prime}\right)$ has a $\operatorname{Bi}\left(m n, p_{t}\right)$ distribution with

$$
p_{t}=(2 t+1) \delta^{\prime 2}>(\log n)^{-3} \delta^{\prime}>n^{-5 / 6} .
$$

The probability that no $r_{i}$ lies in this interval is thus

$$
\left(1-p_{t}\right)^{m n} \leq e^{-m n p_{t}}<e^{-n^{1 / 6}}=o\left(n^{-1}\right) .
$$

Summing over the $O\left(n^{1 / 5}\right)$ values of $t$ shows that $\mathbb{P}\left(E_{5}^{c} \cap E_{1}\right)=o(1)$, and hence that $\mathbb{P}\left(E_{5}\right)=1-o(1)$, completing the proof of the lemma.

From now on we shall assume that the right endpoints $R_{1}, \ldots, R_{m n}$ are given, writing $W_{i}$ for $R_{m i}$. The remaining randomness is given by taking independent random variables $L_{i}, 1 \leq i \leq m n$, uniformly distributed on $\left[0, R_{i}\right]$, to obtain an LCD given by chords $\left\{L_{i}, R_{i}\right\}$ already sorted by their right endpoints. Recall that the graph $G_{1}^{m n}$ is constructed from this LCD by taking an edge from each vertex $i$ to a vertex $k$, where $R_{k-1}<L_{i}<R_{k}$ (taking $R_{0}=0$ ). Thus the graph $G_{m}^{n}$ we shall study is given by taking $m$ edges from each vertex $i, 1 \leq i \leq n$, joining $i$ to $l_{i, j}, 1 \leq j \leq m$, where $l_{i, j}=k$ if $W_{k-1}<L_{m(i-1)+j}<W_{k}$.

Speaking somewhat imprecisely, we would like to forget the $R_{i}$ and consider only the $W_{i}$. The only problem is that the value of $l_{i, j}$ is determined 
by a variable $L_{m(i-1)+j}$ distributed uniformly on $\left[0, R_{m(i-1)+j}\right]$. However, as $W_{i-1}<R_{m(i-1)+j} \leq W_{i}$, if we take $L_{m(i-1)+j}$ uniformly distributed on $\left[0, W_{i}\right]$ we increase the probability that the diameter is large, by increasing the probability of loops at $i$. After making this change we can essentially restrict our attention to the case $m=2$. More precisely, we shall only consider two edges from each vertex $i$, to the vertices $l_{i, 1}$ and $l_{i, 2}$. This change just involves deleting edges from the graph, which again can only increase the diameter.

Fix $m \geq 2$. A precise description of the model we shall use from now on is as follows. Suppose that $n$ distinct real numbers $0<W_{1}<\cdots<W_{n}<1$ are given, and let $w_{i}=W_{i}-W_{i-1}$, taking $W_{0}=0$. We construct a random graph $G\left(W_{1}, \ldots, W_{n}\right)$ by taking two edges from each vertex $i$, joining $i$ to $l_{i, 1}$ and $l_{i, 2}$, where the $l_{i, j}$ are independent with $\mathbb{P}\left(l_{i, j}=k\right)=w_{k} / W_{i}$ for $k \leq i$. Suppose now that $R_{1}, \ldots, R_{m n}$ are obtained by sorting $m n$ independent $M_{2}(0,1)$ random variables, and the random graph $G=G\left(W_{1}, \ldots, W_{n}\right), W_{i}=$ $R_{m i}$, is then constructed. For $m=2$ the random graphs $G$ and $G_{2}^{n}$ have almost the same distribution. More precisely and more generally, for any fixed $m \geq 2$ the random graph $G$ can be coupled with $G_{m}^{n}$ so that $G$ may be obtained from $G_{m}^{n}$ by deleting some edges and adding some loops.

Let $m \geq 2$ and $\epsilon>0$ be fixed. For the rest of the paper we concentrate on the $W_{i}$ rather than the $R_{i}$. We shall assume the $W_{i}$ are given and have the properties $E_{1}, \ldots, E_{5}$ above, and form a random graph $G=G\left(W_{1}, \ldots, W_{n}\right)$ as above, writing $\mathbb{P}_{\mathrm{L}}$ for the corresponding probability measure. To simplify the notation in the following results and proofs, the implicit bound in each occurrence of $o($.$) should be taken as a function of n$ only, not depending on the $W_{i}$ or any other parameters, tending to 0 as $n \rightarrow \infty$. (Of course these implicit functions depend on our fixed parameters $m$ and $\epsilon$; they are functions of $n, m$ and $\epsilon$ which tend to 0 as $n \rightarrow \infty$ with $m$ and $\epsilon$ fixed.) In this light, the results are most simply seen as proving an (implicit) bound on some probability that holds for every sufficiently large $n$, and every sequence $0<$ $W_{1}<\cdots<W_{n}<1$ satisfying the stated conditions. With these conventions we shall show that

$$
\mathbb{P}_{\mathrm{L}}(\operatorname{diam}(G) \leq(1+\epsilon) \log n / \log \log n)=1-o(1)
$$

whenever the $W_{i}$ satisfy $E_{1}, \ldots, E_{5}$. Together with Lemma 7 this is sufficient to prove the upper bound in Theorem 1, namely that for $m \geq 2$ a.e. $G_{m}^{n} \in \mathcal{G}_{m}^{n}$ has $\operatorname{diam}\left(G_{m}^{n}\right) \leq(1+\epsilon) \log n / \log \log n$. 


\section{Getting started}

We shall say that a vertex $i$ is useful if $i \leq n /(\log n)^{5}$ and $w_{i} \geq(\log n)^{2} / n$. As we are assuming $E_{1}, \ldots, E_{5}$, if $n$ is large enough then from $E_{4}$ every $i$ with $i<n^{1 / 5}$ is useful; we shall use this fact several times. Our aim in this section is to prove that the following lemma holds for all sufficiently large $n$ and all $0<W_{1}<\cdots<W_{n}<1$ satisfying the stated conditions.

Lemma 8. Assuming $E_{1}, \ldots, E_{5}$, with $\mathbb{P}_{\mathrm{L}}$ probability $1-o(1)$ every vertex $v$ of $G$ is joined by a (descending) path of length at most $8 \log \log n$ to a useful vertex.

Lemma 8 will be proved in two stages, each of which will be given as a separate lemma. For this section it will be convenient to think of $G$ as a directed graph, directing each edge $i j$ with $i>j$ from $i$ to $j$.

Let $v$ be fixed, and consider the vertices reached from $v$ after $k$ steps. More precisely, let $D_{0}=\{v\}$, and for $k \geq 1$ let $D_{k}$ be the set of those vertices outside $D_{0} \cup \cdots \cup D_{k-1}$ incident with an edge directed from some vertex of $D_{k-1}$. Our first lemma shows that $\left|D_{k}\right|$ is likely to be not much smaller than $2\left|D_{k-1}\right|$, as long as neither $D_{k-1}$ nor $D_{k}$ contains any useful vertices.

Let $n_{k}$ be $\left|D_{k}\right|$ if $D_{0} \cup \cdots \cup D_{k}$ contains no useful vertices, and $\infty$ otherwise.

Lemma 9. For $1 \leq v \leq n, 1 \leq k \leq 8 \log \log n$ and $1 \leq c \leq\left|D_{k-1}\right|$ we have

$$
\mathbb{P}_{\mathrm{L}}\left(n_{k} \leq 2 n_{k-1}-c \mid D_{0}, D_{1}, \ldots, D_{k-1}\right) \leq n^{-3 c / 5+o(1)} .
$$

Proof. Let $t=\left|D_{k-1}\right|$, and let $i_{1}, \ldots, i_{t}$ be the vertices of $D_{k-1}$ in some order. We may assume that no vertex of $D_{0} \cup \cdots \cup D_{k-1}$ is useful as otherwise $n_{k-1}=\infty$ and there is nothing to prove. Thus $n_{k-1}=t$, and for each $s$ we have $i_{s} \geq n^{1 / 5}$.

Suppose that $i_{s}$ sends its first and second edges to $j_{2 s-1}$ and $j_{2 s}$. Then $j_{1}, \ldots, j_{2 t}$ are independent, and for $1 \leq s \leq 2 t$ and $1 \leq j^{\prime} \leq i_{\lceil s / 2\rceil}$ we have

$$
\mathbb{P}_{\mathrm{L}}\left(j_{s}=j^{\prime}\right)=w_{j^{\prime}} / W_{i_{\lceil s / 2\rceil}} .
$$

Now $\left|D_{k}\right|$ is the number of distinct $j_{s}$ which lie outside $D_{0} \cup \cdots \cup D_{k-1}$, a set of at most $2^{k}-1$ vertices none of which is useful. Considering the $j_{s}$ in order, we say that a possible value of $j_{s}$ is repetitive if it coincides with a previous $j_{s^{\prime}}$ which is not useful, or lies in $D_{0} \cup \cdots \cup D_{k-1}$. Recalling that $n_{k}=\infty$ if any vertex in $D_{k}$ is useful, we see that $n_{k}$ is at least $2 n_{k-1}$ minus the number of $s$ for which a repetitive choice is made. For a fixed $s$ the number of possible 
repetitive choices is at most $2^{k+1}$. Consider one such choice $j^{\prime}$. As $j^{\prime}$ is not useful, either $w_{j^{\prime}}<(\log n)^{2} / n$ or $j^{\prime}>n /(\log n)^{5}$. In the first case, using $E_{1}$,

$$
W_{i_{\lceil s / 2\rceil}} \geq W_{\left\lceil n^{1 / 5}\right\rceil} \geq \sqrt{n^{1 / 5} / n} / 2=n^{-2 / 5} / 2,
$$

so

$$
\mathbb{P}_{\mathrm{L}}\left(j_{s}=j^{\prime}\right)=w_{j^{\prime}} / W_{i_{\lceil s / 2\rceil}} \leq n^{-3 / 5+o(1)} .
$$

In the second case we have $w_{j^{\prime}} \leq n^{-4 / 5}$ as $E_{5}$ holds. Also, as $i_{\lceil s / 2\rceil} \geq j^{\prime} \geq n^{4 / 5}$ we have $W_{i_{\lceil s / 2\rceil}} \geq n^{-1 / 10} / 2$, and again $\mathbb{P}_{\mathrm{L}}\left(j_{s}=j^{\prime}\right) \leq n^{-3 / 5+o(1)}$.

Thus at each stage the probability of making a repetitive choice is at most $2^{k+1} n^{-3 / 5+o(1)}$. Since there are only $2\left|D_{k-1}\right|<2^{k}=n^{o(1)}$ stages, the lemma follows.

We shall also need the following simple lemma showing that from any non-useful vertex we have a reasonable chance of hitting a useful vertex. As usual we are assuming that $n$ is sufficiently large.

Lemma 10. Assume $E_{1}, \ldots, E_{5}$ hold and let $i$ be a fixed non-useful vertex. Then the probability that $l_{i, 1}$ is useful is at least $(\log n)^{-3}$.

Proof. Let $i$ be a fixed non-useful vertex. Note that we must have $i \geq n^{1 / 5}$ as $E_{4}$ holds.

Let $2^{b^{\prime}}$ be the largest power of 2 smaller than $\min \left\{i, n /(\log n)^{5}\right\}$. Consider the intervals $I_{t}=\left[2^{t}+1,2^{t+1}\right]$ for $a \leq t<b^{\prime}$, recalling that $s=2^{a}$ is the smallest power of 2 larger than $(\log n)^{7}$. The probability that $l_{i, 1}$ lies in the union of these $I_{t}$ is exactly

$$
\rho=\left(W_{2^{b^{\prime}}}-W_{2^{a}}\right) / W_{i}
$$

As $E_{1}$ holds,

$$
W_{2^{a}} \leq 2 \sqrt{s / n}=n^{-1 / 2+o(1)},
$$

while

$$
W_{i} \geq W_{\left\lceil n^{1 / 5}\right\rceil} \geq n^{-2 / 5} / 2 .
$$

Also, either $i \leq n /(\log n)^{5}$, or $i>n /(\log n)^{5}$. In the first case $2^{b^{\prime}} \geq i / 2$, so, using $E_{1}$ twice, $W_{2^{b^{\prime}}} / W_{i} \geq 1 / 8$. In the second case $2^{b^{\prime}} \geq n /(\log n)^{5} / 2$, so $W_{2^{b^{\prime}}} \geq(\log n)^{-5 / 2} / 2 \geq 100(\log n)^{-3}$. As we always have $W_{i} \leq 1$, in either case $W_{2^{b^{\prime}}} / W_{i} \geq 100(\log n)^{-3}$. Combined with (9) and (10) this shows that $\rho \geq 99(\log n)^{-3}$. 
Let us say that a vertex $i$ is $\operatorname{good}$ if $w_{i} \geq 1 /(10 \sqrt{i n})$. Then $E_{2}$ says exactly that for $a \leq t<b$ each interval $I_{t}$ contains at least $2^{t-1}$ good vertices. As each good vertex $i$ in $I_{t}$ has weight $w_{i} \geq 1 /\left(10 \sqrt{2^{t+1} n}\right)$, the total weight of good vertices within $I_{t}$ is at least $2^{t-1} /\left(10 \sqrt{2^{t+1} n}\right) \geq \sqrt{2^{t} / n} / 30$. On the other hand, the total weight of $I_{t}$ is $W_{2^{t+1}}-W_{2^{t}} \leq W_{2^{t+1}} \leq 3 \sqrt{2^{t} / n}$. For $a \leq t \leq b^{\prime}$, the whole of $I_{t}$ lies to the left of $i$, and the probability that $l_{i, 1}$ is good given that $l_{i, 1} \in I_{t}$ is thus at least $1 / 90$. As $2^{b^{\prime}} \leq n /(\log n)^{5}$, any good vertex of $I_{t}$ is also useful. This shows that the probability that $l_{i, 1}$ is useful is at least $\rho / 90$, from which the lemma follows.

Using the two lemmas above, it is now easy to prove Lemma 8.

Proof of Lemma 8. Fix a vertex $v$, and let $K=\lfloor 8 \log \log n\rfloor-1$. From Lemma 9, with probability at least $1-n^{-6 / 5+o(1)}=1-o\left(n^{-1}\right)$ either $v$ is within distance $K$ of a useful vertex, or we have $\left|D_{k}\right|=2\left|D_{k-1}\right|$ for all but at most one value of $k, 1 \leq k \leq K$, and $\left|D_{k}\right| \geq 2\left|D_{k-1}\right|-1$ for all $k, 1 \leq k \leq K$. Suppose that the second case holds. Then $\left|D_{K}\right| \geq 2^{K-1} \geq 2(\log n)^{4}$, provided $n$ is sufficiently large. Given the sequence $D_{0}, \ldots, D_{K}$, the vertices $l_{i, 1}, i \in D_{K}$, are independent with their original distribution. If $D_{K}$ does not contain a useful vertex then, from Lemma 10, the probability that no $i$ in $D_{K}$ has a useful neighbour is at most

$$
\left(1-(\log n)^{-3}\right)^{\left|D_{K}\right|} \leq \exp \left(-\left|D_{K}\right| /(\log n)^{3}\right) \leq n^{-2} .
$$

Thus with probability at least $1-o\left(n^{-1}\right)$ the given vertex $v$ is within distance $8 \log \log n$ of a useful vertex. As there are only $n$ vertices $v$ to consider, Lemma 8 follows.

\section{Expanding neighbourhoods}

The lemma below gives the heart of the argument. As before, $o($.$) notation$ refers to an implicit function depending on $n$ and $\epsilon$ only. The statement of the lemma then applies for all sufficiently large $n$ and all $0<W_{1}<\cdots<W_{n}<1$ satisfying the stated conditions.

Lemma 11. Assume that $E_{1}, \ldots, E_{5}$ hold, let $\epsilon>0$ be fixed, and let $v$, $1 \leq v \leq n$, be a useful vertex. With $\mathbb{P}_{\mathrm{L}}$ probability $1-o\left(n^{-1}\right)$ there is a path in $G$ between $v$ and 1 of length at most $(1 / 2+\epsilon) \log n / \log \log n$.

The basic strategy of the proof is to divide $[s+1, n]$ into intervals of the form $I_{t}=\left[2^{t}+1,2^{t+1}\right]$. Given the set of vertices $\Gamma_{k}$ at distance $k$ from $v$, we consider the expected size of $\Gamma_{k+1} \cap I_{t}$. Whenever this is bigger than $\log n$ we 
will have enough independence to show that the actual size of $\Gamma_{k+1} \cap I_{t}$ will be close to its expected value. This will show that the weight $f_{k}=\sum_{i \in \Gamma_{k}} 1 / \sqrt{i n}$ almost certainly grows at a certain rate, depending on its current value. In fact we only consider the intervals $I_{t}$ for $a \leq t<b$ where, as before, $s=2^{a}$ is the smallest power of 2 larger than $(\log n)^{7}$, and $2^{b}$ is the largest power of 2 smaller than $2 n / 3$. Thus $a \sim 7 \log _{2} \log n$ and $b \sim \log _{2} n$.

As the proof of Lemma 11 is a little complicated, we state part of the estimation as separate lemma. What this lemma claims is that if the $f_{k}$ satisfy a certain condition then they grow fast enough.

Lemma 12. Suppose that $\epsilon>0$, and set $K=(1 / 2+\epsilon) \log n / \log \log n-1$. Let $f_{0}, f_{1}, \ldots$ be a sequence of real numbers with $f_{0} \geq(\log n)^{2} / n$ and

$$
f_{k+1} \geq \min \left\{2 \log _{2}\left(f_{k} n / \log n\right)-31, b-a-1\right\} f_{k} / 1000,
$$

for $k \geq 0$. Then, provided $n$ is sufficiently large, $\ell=\min \left\{k: f_{k} \geq(\log n)^{2} / \sqrt{n}\right\}$ exists and is at most $K$.

Proof. Provided $n$ is sufficiently large we have $\log _{2}\left(f_{0} n / \log n\right) \geq$ $\log _{2}(\log n) \geq 1016$. Thus (11) implies that $f_{1} \geq 2 f_{0}$. It follows inductively that $f_{k+1} \geq 2 f_{k}$ and $f_{k} \geq 2^{k} f_{0}$ hold for all $k \geq 0$. This shows that $\ell$ exists.

As $b-a-1 \geq \log _{2} n-8 \log _{2} \log n$, the minimum in (11) is different from the first term only when $f_{k} \geq(\log n)^{-3} / \sqrt{n}$. If this first happens at $k=k_{0}$, say, then for $k \geq k_{0}$ we have

$$
f_{k+1} \geq(b-a-1) f_{k} / 1000=\left(\log _{2} n\right)^{1+o(1)} f_{k},
$$

which implies that $\ell \leq k_{0}+6$. Thus

$$
f_{k+1} \geq \frac{\log _{2}\left(f_{k} n / \log n\right)-16}{500} f_{k}
$$

for $0 \leq k<\ell-6$. As $f_{k} \geq 2^{k} f_{0}$ and $\log _{2}\left(f_{0} n / \log n\right) \geq 17$ (if $n$ is large enough), we have $\log _{2}\left(f_{k} n / \log n\right) \geq k+17$. Combined with (12) this implies that $f_{k+1} \geq f_{k}(k+1) / 500$ for $0 \leq k<\ell-6$, and hence that

$$
f_{\ell-6} \geq \frac{(\ell-6) !}{500^{\ell-6}} f_{0} \geq\left(\frac{\ell-6}{500 e}\right)^{\ell-6} f_{0}
$$

using Stirling's formula. As $f_{\ell-6}<(\log n)^{2} / \sqrt{n} \leq \sqrt{n} f_{0}$ this implies that $\ell-6 \leq(1 / 2+\epsilon / 2) \log n / \log \log n<K-6$, and the lemma follows.

With this straightforward but slightly messy calculation behind us, we turn to the proof of Lemma 11. 
Proof of Lemma 11. Roughly speaking, we consider the set of vertices within distance $k$ of $v$, showing that the weight of this set increases rapidly with $k$. In fact, to keep independence, when working outwards from $v$ we only allow ourselves to use an edge $i j$ from a vertex $i$ at distance $k$ to a new vertex $j$ if $i>j$ and $i j$ is the first edge from $i$ or if $i<j$ and $i j$ is the second edge from $j$. As before we say that a vertex $i$ is good if

$$
w_{i} \geq \frac{1}{10 \sqrt{i n}} .
$$

Let $\Gamma_{0}=\{v\}$, and for $k \geq 1$ let $\Gamma_{k}$ consist of those $j$ in $\left[s+1,2^{b}\right] \backslash\left(\Gamma_{0} \cup\right.$ $\left.\cdots \cup \Gamma_{k-1}\right)$ such that $j$ is good and either $l_{j, 2} \in \Gamma_{k-1}$ or there is an $i \in \Gamma_{k-1}$ with $l_{i, 1}=j$. We shall write $N_{k}$ for $\Gamma_{0} \cup \cdots \cup \Gamma_{k}$.

Rather than the actual weight (sum of the $w_{i}$ ) we consider the 'ideal weight' of $\Gamma_{k}$, given by

$$
f_{k}=\sum_{i \in \Gamma_{k}} \frac{1}{\sqrt{i n}}
$$

Note that for $k \geq 1$ the set $\Gamma_{k}$ contains only good vertices, so its actual weight is at least $f_{k} / 10$. For the sake of simplicity we shall assume that $v$ itself is good, as well as useful. The case that $v$ is useful but not good can be dealt with by redefining $f_{0}$ to be $(\log n)^{2} / n$. It is easy to check that the first step in the argument that follows requires only that $1 / \sqrt{v n} \geq f_{0}$ and that $w_{v} \geq f_{0} / 10$. At the end of the argument we only use $f_{0} \geq(\log n)^{2} / n$, and nowhere else is the goodness of $v$ relevant.

Given $\Gamma_{0}, \ldots, \Gamma_{k}$, for each $i \in \Gamma_{k}$ the random variable $l_{i, 1}$ has its original unconditioned distribution, since we have 'looked at' $l_{i, 2}$ but not $l_{i, 1}$. Similarly, for $i \notin N_{k}$ all we know about $l_{i, 2}$ is that $l_{i, 2} \notin N_{k-1}$, and the variables $\left\{l_{i, 1}: i \in \Gamma_{k}\right\} \cup\left\{l_{i, 2}: i \notin N_{k}\right\}$ are independent.

Let us fix a $t$ with $a \leq t<b$, and consider the interval $I_{t}=\left[2^{t}+1,2^{t+1}\right]$. Let $X$ be the number of vertices of $N_{k} \cap I_{t}$ (these vertices are 'excluded' for our present purpose), and let $S$ be the set of good vertices of $I_{t} \backslash N_{k}$. Now $E_{2}$ states exactly that $I_{t}$ contains at least $2^{t-1}$ good vertices, so $|S| \geq 2^{t-1}-X$. Let $i_{1}, \ldots, i_{d}$ be the vertices of $\Gamma_{k} \cap\left[2^{t+1}+1, n\right]$, listed in any order. We consider examining each $i$ in turn to see whether $l_{i, 1}$ lies in $S$. More precisely, let $C_{r}$ be the number of distinct elements of $S$ equal to $l_{i, 1}$ for $i \in\left\{i_{1}, \ldots, i_{r}\right\}$, so $\left|\Gamma_{k+1} \cap I_{t}\right| \geq C_{d}$. Conditioning on everything so far, i.e., on $\Gamma_{0}, \ldots, \Gamma_{k}$ and $l_{i_{1}, 1}, \ldots, l_{i_{r-1}, 1}$, provided $C_{r-1} \leq 2^{t-2}-X$ the probability $P_{r}$ that $C_{r}=C_{r-1}+1$ satisfies

$$
P_{r} \geq 2^{t-2} \frac{1}{10 \sqrt{2^{t+1} n}} W_{i_{r}}^{-1}
$$


as there are at least $|S|-C_{r-1} \geq 2^{t-2}$ vertices $j$ of $S$ unused, each of weight $w_{j} \geq \frac{1}{10 \sqrt{j n}} \geq \frac{1}{10 \sqrt{2^{t+1} n}}$. Using $W_{i} \leq \sqrt{2 i / n}$ for $i \geq s$,

$$
P_{r} \geq \frac{2^{t-2}}{20 \sqrt{2^{t} n}} \sqrt{\frac{n}{i_{r}}}=\frac{\sqrt{2^{t}}}{80 \sqrt{i_{r}}}=p_{r},
$$

say. Let $Y$ be a random variable given by the sum of $d$ independent Bernoulli random variables $\operatorname{Bi}\left(1, p_{r}\right)$ with means $p_{1}, \ldots, p_{d}$. Then $C_{d}$ stochastically dominates $\min \left\{Y, 2^{t-2}-X\right\}$. (Roughly speaking, (13) implies by induction on $r$ that $C_{r} \geq \sum_{r^{\prime}=1}^{r} \operatorname{Bi}\left(1, p_{r^{\prime}}\right)$ until $C_{r}=2^{t-2}-X$.)

Let

$$
\mu_{1}=\sum_{i \in \Gamma_{k}, i>2^{t+1}} \frac{\sqrt{2^{t}}}{80 \sqrt{i}}=\sum_{r=1}^{d} p_{r} .
$$

Provided $\mu_{1} \geq 10 \log n$ we have from Lemma 6 that $\mathbb{P}\left(Y \leq \mu_{1} / 2\right) \leq n^{-5 / 4}$, and hence that

$$
\mathbb{P}_{\mathrm{L}}\left(C_{d} \leq \min \left\{\mu_{1} / 2,2^{t-2}-X\right\} \mid \Gamma_{0}, \ldots, \Gamma_{k}\right) \leq n^{-5 / 4} .
$$

In other words, if $\mu_{1}$ is large enough, then $\mu_{1} / 2$ vertices of $I_{t}$ are likely to be hit by edges coming out of $\Gamma_{k} \cap\left[2^{t+1}+1, n\right]$, unless this would mean that more than $2^{t-2}$ vertices of $I_{t}$ would be contained in $N_{k+1}$.

We now consider edges from $I_{t}$ into $\Gamma_{k} \cap\left[s+1,2^{t}\right]$.

Let $X^{\prime}=X+C_{d}$ and let $S^{\prime}$ be the set of good vertices of $I_{t} \backslash N_{k}$ not counted in $C_{d}$, so $\left|S^{\prime}\right| \geq 2^{t-1}-X^{\prime}$. We consider the number $C^{\prime}$ of $j \in S^{\prime}$ for which $l_{j, 2}$ lies in $\Gamma_{k}$, noting that $\left|\Gamma_{k+1} \cap I_{t}\right| \geq C^{\prime}$. Given $\Gamma_{0}, \ldots, \Gamma_{k}$, for each $j \in S^{\prime}$ the random variable $l_{j, 2}$ has its original distribution conditioned on $l_{j, 2} \notin N_{k-1}$. Thus, as $\Gamma_{k}$ is disjoint from $N_{k-1}$,

$$
\begin{aligned}
\mathbb{P}\left(l_{j, 2} \in \Gamma_{k}\right) & \geq \sum_{i \in \Gamma_{k}, i \leq j} w_{i} / W_{j} \\
& \geq \sum_{i \in \Gamma_{k}, i \leq 2^{t}} w_{i} / W_{2^{t+1}} \\
& \geq \sum_{i \in \Gamma_{k}, i \leq 2^{t}} \frac{1}{10 \sqrt{i n}} \sqrt{\frac{n}{2^{t+2}}} \\
& =\sum_{i \in \Gamma_{k}, i \leq 2^{t}} \frac{1}{20 \sqrt{2^{t} i}}=p
\end{aligned}
$$

say. As the $l_{j, 2}$ are independent, the number $C^{\prime}$ of $j \in S^{\prime}$ for which $l_{j, 2}$ is in $\Gamma_{k}$ stochastically dominates a $\operatorname{Bi}\left(2^{t-1}-X^{\prime}, p\right)$ distribution. Let $\mu_{2}=2^{t-2} p$. 
Then provided $\mu_{2} \geq 10 \log n$ we have from Lemma 6 that

$$
\mathbb{P}_{\mathrm{L}}\left(C^{\prime} \leq \mu_{2} / 2 \mid \Gamma_{0}, \ldots, \Gamma_{k}, X^{\prime}<2^{t-2}\right) \leq n^{-5 / 4} .
$$

In other words, if $\mu_{2} \geq 10 \log n$ and we have not yet (in $N_{k}$ together with the part of $\Gamma_{k+1}$ we found above) reached $2^{t-2}$ vertices of $I_{t}$, then it is likely that $\mu_{2} / 2$ new vertices of $I_{t}$ can be reached by following back edges from $\Gamma_{k} \cap\left[s+1,2^{t}\right]$.

We claim that

$$
\mathbb{P}_{\mathrm{L}}\left(\left|\Gamma_{k+1} \cap I_{t}\right| \leq \min \left\{\frac{\mu_{1}+\mu_{2}}{4}, 2^{t-2}-\left|N_{k} \cap I_{t}\right|\right\} \mid \Gamma_{0}, \ldots, \Gamma_{k}\right) \leq n^{-5 / 4}
$$

holds whenever $\mu_{1}+\mu_{2} \geq 20 \log n$. To see this, suppose first that $\mu_{1} \geq \mu_{2}$. Then $\mu_{1} \geq\left(\mu_{1}+\mu_{2}\right) / 2$ and $\mu_{1} \geq 10 \log n$. As $X=\left|N_{k} \cap I_{t}\right|$ by definition, while $\left|\Gamma_{k+1} \cap I_{t}\right| \geq C_{d}$, in this case (16) follows from (14).

Suppose instead that $\mu_{2}>\mu_{1}$. Then $\mu_{2} \geq\left(\mu_{1}+\mu_{2}\right) / 2$ and $\mu_{2} \geq 10 \log n$. Thus (15) implies that

$$
\mathbb{P}_{\mathrm{L}}\left(C^{\prime} \leq\left(\mu_{1}+\mu_{2}\right) / 4 \mid \Gamma_{0}, \ldots, \Gamma_{k}, X^{\prime}<2^{t-2}\right) \leq n^{-5 / 4} .
$$

Now $\left|\Gamma_{k+1} \cap I_{t}\right| \geq C^{\prime}$. Also, $X^{\prime} \leq\left|\Gamma_{k+1} \cap I_{t}\right|+\left|N_{k} \cap I_{t}\right|$. Thus if $X^{\prime} \geq 2^{t-2}$ then $\left|\Gamma_{k+1} \cap I_{t}\right| \geq 2^{t-2}-\left|N_{k} \cap I_{t}\right|$. Thus (17) implies (16) in this case also, showing that (16) holds whenever $\mu_{1}+\mu_{2} \geq 20 \log n$.

We shall now vary $t$ in the range $a \leq t<b$ to obtain a lower bound on the weight of $\Gamma_{k+1}$. Note that

$$
\mu_{1}+\mu_{2}=\mu_{1}(t)+\mu_{2}(t)=\frac{\sqrt{2^{t}}}{80} \sum_{i \in \Gamma_{k} \backslash I_{t}} \frac{1}{\sqrt{i}} .
$$

Recalling that

$$
f_{k}=\frac{1}{\sqrt{n}} \sum_{i \in \Gamma_{k}} \frac{1}{\sqrt{i}}
$$

it follows that for all but at most one value of $t$ we have

$$
\mu_{1}+\mu_{2} \geq \frac{\sqrt{2^{t} n}}{160} f_{k}
$$

and the proof is nearly complete. 
Given $\Gamma_{0}, \ldots, \Gamma_{k}$, let $\mathcal{T}$ be the set of indices $t, a \leq t<b$, for which $\sqrt{2^{t} n} f_{k} / 160 \geq 20 \log n$. As $2^{b} \geq n / 3$, considering the smallest element of $\mathcal{T}$ shows that

$$
|\mathcal{T}| \geq \min \left\{2 \log _{2}\left(f_{k} n / \log n\right)-30, b-a\right\} .
$$

Let us write $\mathcal{T}^{\prime}$ for the set of $t \in \mathcal{T}$ for which the quantity $\mu_{1}+\mu_{2}$ defined above (which depends on $t$ ) is at least $\sqrt{2^{t} n} f_{k} / 160$, so $\left|\mathcal{T}^{\prime}\right| \geq|\mathcal{T}|-1$. We shall say that an interval $I_{t}$ is full at stage $k+1$ if $\left|N_{k+1} \cap I_{t}\right| \geq 2^{t-2}$. From (16), for each $t \in \mathcal{T}^{\prime}$ with probability $1-O\left(n^{-5 / 4}\right)$ either $I_{t}$ is full in $N_{k+1}$ or $\left|\Gamma_{k+1} \cap I_{t}\right| \geq \sqrt{2^{t} n} f_{k} / 640$. In the latter case we have

$$
\sum_{i \in \Gamma_{k+1} \cap I_{t}} \frac{1}{\sqrt{i n}} \geq \frac{\sqrt{2^{t} n} f_{k}}{640 \sqrt{2^{t+1} n}} \geq \frac{f_{k}}{1000} .
$$

Thus, with probability $1-O\left((\log n) n^{-5 / 4}\right)=1-O\left(n^{-6 / 5}\right)$ either some $I_{t}$ is full at stage $k+1$, or

$$
f_{k+1} \geq \frac{\left|\mathcal{T}^{\prime}\right| f_{k}}{1000} \geq \frac{\min \left\{2 \log _{2}\left(f_{k} n / \log n\right)-31, b-a-1\right\}}{1000} f_{k} .
$$

Starting with $\Gamma_{0}=\{v\}$ let us construct $\Gamma_{1}, \Gamma_{2}, \ldots$, and let $K$ be the minimum $k$ for which either $f_{k} \geq(\log n)^{2} / \sqrt{n}$ or (18) does not hold. As $v$ is useful we have $f_{0}=w_{v} \geq(\log n)^{2} / n$. As (18) holds for $0 \leq k<K$, Lemma 12 implies that $K \leq(1 / 2+\epsilon) \log n / \log \log n-1$.

We claim that

$$
f_{K} \geq(\log n)^{2} / \sqrt{n}
$$

holds with probability $1-o\left(n^{-1}\right)$. The only possible problem is that some $I_{t}$ may become full at some stage.

Suppose first that some $I_{t}, a \leq t<b$, is full at some stage $k, 1 \leq k \leq K$. Then (leaving out $\Gamma_{0}$ as $v$ is not necessarily good), in $N_{K} \backslash\{v\}$ we have at least $2^{t-2}-1$ good vertices in $I_{t}$. This gives

$$
f_{1}+\cdots+f_{K} \geq \frac{2^{t-2}-1}{10 \sqrt{2^{t+1} n}} \geq \frac{\sqrt{2^{t}}}{160 \sqrt{n}} \geq \frac{\sqrt{2^{a}}}{160 \sqrt{n}}=\frac{\sqrt{s}}{160 \sqrt{n}}>\frac{(\log n)^{3}}{\sqrt{n}} .
$$

As $K<\log n$ and $f_{k}<(\log n)^{2} / \sqrt{n}$ for $k<K$, this implies (19). On the other hand, if no $I_{t}$ becomes full at any stage, then (18) holds with probability at least $1-O\left(n^{-6 / 5}\right)$ at each of $K \leq \log n$ stages, so (19) holds with probability $1-o\left(n^{-1}\right)$, as claimed.

If (19) holds we stop the construction of the sequence $\Gamma_{1}, \Gamma_{2}, \ldots$ at $\Gamma_{K}$. Given the sequence so far, the $l_{i, 1}, i \in \Gamma_{K}$, are independent. As $K>0$ we 
have $\Gamma_{K} \subset[s+1, n]$ by construction. Thus, since $E_{1}$ and $E_{3}$ hold, for each $i \in \Gamma_{K}$ we have

$$
\mathbb{P}_{\mathrm{L}}\left(l_{i, 1}=1 \mid \Gamma_{0}, \ldots, \Gamma_{K}\right)=\frac{w_{1}}{W_{i}} \geq \frac{4}{\log n \sqrt{n}} \sqrt{\frac{n}{2 i}}>\frac{2}{\log n \sqrt{i}}
$$

Conditioning on the sequence $\Gamma_{0}, \ldots, \Gamma_{K}$, if (19) holds the probability that $\Gamma_{K}$ does not send an edge to vertex 1 is at most

$$
\prod_{i \in \Gamma_{K}}\left(1-\frac{2}{\log n \sqrt{i}}\right) \leq \exp \left(-\sum_{i \in \Gamma_{K}} \frac{2}{\log n \sqrt{i}}\right)=\exp \left(-\frac{2 f_{K} \sqrt{n}}{\log n}\right) \leq n^{-2}
$$

Since (19) holds with probability $1-o\left(n^{-1}\right)$, it follows that with probability $1-o\left(n^{-1}\right)$ the vertex $v$ is connected to 1 by a path of length at most $K+1 \leq$ $(1 / 2+\epsilon) \log n / \log \log n$, completing the proof of Lemma 11 .

Combining the results above proves Theorem 1.

Proof of Theorem 1. We have already proved the lower bound as Theorem 5. Fix $m \geq 2$ and $\epsilon>0$. Together Lemmas 8 and 11, applied with $\epsilon / 4$ in place of $\epsilon$, imply that given $W_{1}, \ldots, W_{n}$

$\mathbb{P}_{\mathrm{L}}\left(\operatorname{diam}\left(G\left(W_{1}, \ldots, W_{n}\right)\right)>(1+\epsilon / 2) \log n / \log \log n+16 \log \log n\right)=o(1)$,

whenever $E_{1}, \ldots, E_{5}$ hold. Let $R_{1}, \ldots, R_{m n}$ be obtained by sorting $m n$ independent $M_{2}(0,1)$ random variables, set $W_{i}=R_{m i}$, and consider the graph $G=G\left(W_{1}, \ldots, W_{n}\right)$. As each $E_{i}$ holds with probability $1-o(1)$ by Lemma 7 , it follows that

$$
\mathbb{P}(\operatorname{diam}(G)>(1+\epsilon / 2) \log n / \log \log n+16 \log \log n)=o(1) .
$$

Finally, we have shown at the end of section 6 that the random graph $G$ defined in this way may be coupled with $G_{m}^{n}$ so that $G$ can be obtained from $G_{m}^{n}$ by (perhaps) deleting some edges and adding some loops. Under this coupling $\operatorname{diam}(G) \geq \operatorname{diam}\left(G_{m}^{n}\right)$, completing the proof.

We next turn briefly to the case $m=1$, which has been studied earlier and behaves rather differently. 


\section{The case $m=1$}

One particular step in the proof of the upper bound on $\operatorname{diam}\left(G_{m}^{n}\right)$ given above appears at first sight to be rather wasteful, namely the step where we split the edges into two separate classes, considering at every point only edges from one class. Something like this is necessary to achieve independence when considering how the $(k+1)$-neighbourhood $N_{k+1}$ of a given vertex relates to $N_{k}$. However, as the neighbourhoods grow quite quickly, one would imagine that we only rarely try to use edges already used. This suggests that a version of the proof above should apply with $m=1$, losing about a factor of two in the growth rate, which would have negligible effect on the diameter. This turns out to be not the case - for $m=1$ the diameter is in fact $\Theta(\log n)$, as shown by Pittel [24] in a slightly different context described below.

For $m=1$ the graph $G_{m}^{n}$ has a rather simple structure. Each vertex sends an edge either to itself or to an earlier vertex, so the graph consists of components which are trees, each with a loop attached. It is much more natural to consider trees rather than forests. Trees on $\{1,2, \ldots, n\}$ where each vertex other than the first sends an edge to an earlier vertex are known as recursive trees; random recursive trees have been studied for some time, see, for example, [22]. When a random recursive tree is constructed one vertex at a time, each new vertex must be joined to an earlier vertex chosen according to some rule. The most studied case is that of a uniform choice, but probabilities essentially proportional to degrees have also been considered. Such objects (where for the first vertex degree plus one is used) are known as random plane-oriented recursive trees, see [27,21], for example. Pittel [24] showed that the height (maximum distance of a vertex from the root) of such an object is $(c+o(1)) \log n$ with probability $1-o(1)$, where $c=(2 \gamma)^{-1}$ for $\gamma$ the solution of $\gamma e^{1+\gamma}=1$. It is easy to see that his method also shows that the diameter is $(2 c+o(1)) \log n$. Now $G_{1}^{n}$ has on average $\Theta(\log n)$ loops. Thus with high probability $G_{1}^{n}$ has $O(\log n)$ loops and hence $O(\log n)$ components. It is easy to see that Pittel's method can be applied to prove the following result.

Theorem 13. Let $\gamma$ be the solution of $\gamma e^{1+\gamma}=1$, and let $\epsilon>0$ be fixed. Then for almost every $G_{1}^{n} \in \mathcal{G}_{1}^{n}$ the largest distance between two vertices in the same component is between $\left(\gamma^{-1}-\epsilon\right) \log n$ and $\left(\gamma^{-1}+\epsilon\right) \log n$.

Note that this result is given only for completeness - for $m=1$ the random plane-oriented recursive tree model is actually more natural than $G_{1}^{n}$. 


\section{Conclusions}

In several papers random graphs modeling complex real-world networks are studied, in particular with respect to their diameter or average diameter, the average distance between two vertices. One model studied is the process $G_{m}^{n}$ considered here. Another model is given by taking a function $P(d), d \geq 1$, to represent the probability that a vertex has degree $d$, and choosing a graph on $n$ vertices at random from all graphs with this distribution of degrees.

In $[1,5,23]$ results from computer experiments have been published suggesting that for the second model the diameter of the random graph will vary as $A+B \log n$ when $n$ is large, for a wide variety of functions $P(d)$. In [23] a heuristic argument is given, based on the standard neighbourhood expansion method; roughly speaking, if one follows a random edge to one of its endvertices $v$, the probability $P^{\prime}(d)$ that $v$ will have degree $d$ should be proportional to $d P(d)$. Thus, writing $N_{k}(v)$ for the set of vertices within distance $k$ of a given vertex $v$, one would expect $\left|N_{k+1}(v)\right|$ to be about

$$
f=\sum d P^{\prime}(d)=\left(\sum d^{2} P(d)\right) /\left(\sum d P(d)\right)
$$

times larger than $\left|N_{k}(v)\right|$. One then expects the diameter of the graph to be about $\log n / \log f$.

In the context of $G_{m}^{n}$, which has $P(d)$ proportional to $d^{-3}$, this argument actually predicts an expansion factor of $\log n$, and hence a diameter of around $\log n / \log \log n$. The same expansion factor is predicted by approximating $G_{m}^{n}$ by a graph in which each edge $i j$ is present with probability proportional to $1 / \sqrt{i j}$, as in the proof of Theorem 5 . As shown by Theorem 1 , this prediction is correct if $m \geq 2$. The explanation for the prediction of $\Theta(\log n)$ given by the more sophisticated heuristic in [23] is that a cutoff is imposed on the degree distribution so that the formalism used there applies. In the light of our results, we believe that the heuristic given above will apply in many situations, in particular when the graph is chosen at random subject to a power law distribution being imposed on the degrees. On the other hand, in any given case it can be highly non-trivial to take the actual structure into account. Furthermore, this structure can cause the heuristic argument to fail, as shown by the case of $G_{1}^{n}$, for which exactly the same heuristics apply as for $G_{2}^{n}$.

We finish with two more remarks concerning possible extensions of Theorem 1 . For the first, note that the upper bound on the diameter of $G_{m}^{n}$ given by Theorem 1 is a pure existence result. An interesting question is whether a short path between two given vertices can be constructed quickly using only 'local' information (see [20], for example). In $G_{m}^{n}$ we believe that it will 
take $\Theta(\log n)$ steps to reach vertex 1 from vertex $n$ using local information. In the other direction, given any set $S$ of $o(n)$ vertices of a typical $G_{m}^{n-1}$ it is easy to check that the probability of vertex $n$ sending an edge to $S$ is $o(1)$. It follows that at least $\Theta(n)$ steps are needed to locally construct a path from 1 to $n$ in $G_{m}^{n}$.

The second remark concerns the 'robustness' of the graph $G_{m}^{n}$. In [2] the following question is raised, and answered experimentally: how many vertices can be deleted at random from a 'scale-free' random graph without the diameter increasing too much, ignoring small components if necessary? It is easy to see that the expanding neighbourhoods argument of section 8 is unaffected if the vertices of $G_{m}^{n}$ are deleted independently with probability $p$, for any constant $p<1$. In the terminology of section 7 , from any 'useful' vertex there will be a short path to the first surviving vertex of $G_{m}^{n}$. In general, after deleting vertices in this manner, what remains of $G_{m}^{n}$ will consist of many small components, together with a giant component whose diameter is asymptotically $\log n / \log \log n$, for any constant $p<1$.

Finally, we finish by mentioning an interesting problem to which one of the referees has drawn our attention. Suppose we are given a rooted tree of height $n$ in which each non-leaf node has $k \geq 2$ children, and suppose that the edges are weighted with independent standard exponential random variables. What is the asymptotic distribution of the minimum weight of a path from root to leaf as $n \rightarrow \infty$ with $k$ fixed? For results on this question, related to the question of which vertices of $G_{m}^{n}$ can be reached from an initial vertex by descending paths of a certain length, see [16,25].

\section{References}

[1] R. Albert, H. Jeong and A.-L. Barabási: Diameter of the world-wide web, Nature 401 (1999), 130-131.

[2] R. Albert, H. JeOng and A.-L. Barabási: Error and attack tolerance of complex networks, Nature 406 (2000), 378-382.

[3] A.-L. BARABÁsi and R. Albert: Emergence of scaling in random networks, Science 286 (1999), 509-512.

[4] A.-L. Barabási, R. Albert and H. JeOng: Mean-field theory for scale-free random networks, Physica A 272 (1999), 173-187.

[5] A.-L. Barabási, R. Albert and H. Jeong: Scale-free characteristics of random networks: the topology of the world-wide web, Physica A 281 (2000), 69-77.

[6] B. BollobÁs: The diameter of random graphs, Trans. Amer. Math. Soc. 267 (1981), $41-52$.

[7] B. Bollobás: Random Graphs, Second Edition, Cambridge studies in advanced mathematics, vol. 73, Cambridge University Press, Cambridge, 2001, xviii +498 pp.

[8] B. BollobÁs and F. R. K. Chung: The diameter of a cycle plus a random matching, SIAM J. Discrete Math. 1 (1988), 328-333. 
[9] B. BollobÁs and W. Fernandez DE LA VEGA: The diameter of random regular graphs, Combinatorica 2 (1982), 125-134.

[10] B. Bollobás and V. KleE: Diameters of random bipartite graphs, Combinatorica 4 (1984), 7-19.

[11] B. BollobÁs and O. M. Riondan: Linearized chord diagrams and an upper bound for Vassiliev invariants, J. Knot Theory Ramifications 9 (2000), 847-853.

[12] B. Bollobás, O. M. Riordan, J. Spencer and G. Tusnády: The degree sequence of a scale-free random graph process, Random Structures and Algorithms 18 (2001), 279-290.

[13] Ju. D. Burtin: Asymptotic estimates of the diameter and the independence and domination numbers of a random graph, Dokl. Akad. Nauk SSSR 209 (1973), 765768, translated in Soviet Math. Dokl. 14 (1973), 497-501.

[14] Ju. D. BuRTin: Extremal metric characteristics of a random graph. I, Teor. Verojatnost. i Primenen. 19 (1974), 740-754.

[15] H. Chernoff: A measure of asymptotic efficiency for tests of a hypothesis based on the sum of observations, Ann. Math. Statistics 23 (1952) 493-507.

[16] L. Devroye: Branching processes and their applications in the analysis of tree structures and tree algorithms, in Probabilistic methods for algorithmic discrete mathematics, Algorithms Combin. 16 (1998), 249-314.

[17] S. Janson: On concentration of probability, in Contemporary Combinatorics, Bolyai Soc. Math. Stud. 10, B. Bollobás ed., pp. 289-301.

[18] V. Klee and D. G. Larman: Diameters of random graphs, Canad. J. Math. 33 (1981), 618-640.

[19] V. Klee, D. G. Larman and E. M. Wright: The diameter of almost all bipartite graphs, Studia Sci. Math. Hungar. 15 (1980), 39-43.

[20] J. KLEINBERG: The small-world phenomenon: an algorithmic perspective, Cornell Computer Science Technical Report 99-1776 (October 1999).

[21] H. M. Mahmoud, R. T. Smythe and J. Szymański: On the structure of random plane-oriented recursive trees and their branches, Random Structures and Algorithms 4 (1993), 151-176.

[22] H. M. Mahmoud and R. T. Smythe: A survey of recursive trees, Th. of Probability and Math. Statistics 51 (1995), 1-27.

[23] M. E. J. Newman, S. H. Strogatz and D. J. Watts: Random graphs with arbitrary degree distribution and their applications, Physical Review E 64 (2001), 026118.

[24] B. PitTel: Note on the heights of random recursive trees and random $m$-ary search trees, Random Structures and Algorithms 5 (1994), 337-347.

[25] P. RÉvÉsz: Critical branching Wiener process in $\mathbb{R}^{d}$, in Random walks (Budapest, 1998), Bolyai Soc. Math. Stud. 9 (1999), 299-348.

[26] A. Stoimenow: Enumeration of chord diagrams and an upper bound for Vassiliev invariants, J. Knot Theory Ramifications 7 (1998), 93-114.

[27] J. Szymański: On a nonuniform random recursive tree, Annals of Discrete Math. 33 (1987), 297-306.

[28] D. J. Watts and S. H. Strogatz: Collective dynamics of 'small-world' networks, Nature 393 (1998), 440-442. 
34 BOLLOBÁS, RIORDAN: THE DIAMETER OF A SCALE-FREE RANDOM GRAPH

Béla Bollobás

Department of Mathematical Sciences University of Memphis

Memphis TN 38152

USA

and

Trinity College

Cambridge CB2 $1 T Q$

$U K$

bollobas@msci.memphis.edu
Oliver Riordan

Trinity College

Cambridge CB2 $1 T Q$

$U K$

omr10@dpmms . cam.ac.uk 